Full text access provided via ACS AuthorChoice

\title{
Communication
}

\section{Unprecedented ultralow detection limit of amines using a thiadiazole-functionalized Zr(IV)-based metal-organic framework}

Arijit Mallick, Ahmed M. El-Zohry, Osama Shekhah, Jun Yin, jiangtao jia, Himanshu Aggarwal, Abdul-Hamid Emwas, Omar F. Mohammed, and Mohamed Eddaoudi

J. Am. Chem. Soc., Just Accepted Manuscript • DOI: 10.1021/jacs.9b01839 • Publication Date (Web): 15 Apr 2019

Downloaded from http://pubs.acs.org on April 21, 2019

\section{Just Accepted}

"Just Accepted" manuscripts have been peer-reviewed and accepted for publication. They are posted online prior to technical editing, formatting for publication and author proofing. The American Chemical Society provides "Just Accepted" as a service to the research community to expedite the dissemination of scientific material as soon as possible after acceptance. "Just Accepted" manuscripts appear in full in PDF format accompanied by an HTML abstract. "Just Accepted" manuscripts have been fully peer reviewed, but should not be considered the official version of record. They are citable by the Digital Object Identifier (DOI®). "Just Accepted" is an optional service offered to authors. Therefore, the "Just Accepted" Web site may not include all articles that will be published in the journal. After a manuscript is technically edited and formatted, it will be removed from the "Just Accepted" Web site and published as an ASAP article. Note that technical editing may introduce minor changes to the manuscript text and/or graphics which could affect content, and all legal disclaimers and ethical guidelines that apply to the journal pertain. ACS cannot be held responsible for errors or consequences arising from the use of information contained in these "Just Accepted" manuscripts. 


\section{Unprecedented Ultralow Detection Limit of Amines Using a Thiadiazole-Functionalized Zr(IV)-Based Metal-Organic} Framework

Arijit Mallick ${ }^{\mathrm{a}}$, Ahmed M. El-Zohryb, Osama Shekhah ${ }^{\mathrm{a}}$, Jun Yin ${ }^{\mathrm{b}}$, Jiangtao Jia ${ }^{\mathrm{a}}$, Himanshu Aggarwal ${ }^{\mathrm{a}}$, Abdul-Hamid Emwas, ${ }^{\mathrm{c}}$ Omar F. Mohammed, ${ }^{\mathrm{b},{ }^{*}}$ and Mohamed Eddaoudia, ${ }^{*}$

${ }^{a}$ King Abdullah University of Science and Technology (KAUST), Functional Materials Design, Discovery and Development Research Group (FMD ${ }^{3}$ ), Advanced Membranes and Porous Materials Center (AMPMC), Division of Physical Sciences and Engineering (PSE), Thuwal 23955-6900, Saudi Arabia.

${ }^{\mathrm{b}}$ King Abdullah University of Science and Technology (KAUST), Division of Physical Sciences and Engineering (PSE), Thuwal 23955-6900, Saudi Arabia.

${ }^{c}$ King Abdullah University of Science and Technology (KAUST), Core Labs, Thuwal 23955-6900, Saudi Arabia. Supporting Information Placeholder

\begin{abstract}
A luminescent Zr(IV)-based MOF, with the underlying feu topology, encompassing a $\pi$-conjugated organic ligand with a thiadiazole functionality exhibits an unprecedented low detection limit of $66 \mathrm{nM}$ for amines in aqueous solution. Markedly, this ultralow detection is driven by hydrogen bonding interactions between the linker and the hosted amines. This observation is supported by Density Functional Theory (DFT) calculations which clearly corroborate the suppression of the twisting motion of thiadiazole core in the presence of amine, reducing significantly the non-radiative recombination pathways and subsequently enhancing the emission intensity. Credibly, nicotine regarded as a harmful chemical and bearing an amine pending group is also detected with high sensitivity, positioning this MOF as a potential sensor for practical environmental applications. This finding serves also as a benchmark to understand the sensing mechanism in MOFs.
\end{abstract}

The need to sense dangerous small molecules at a very low detection limit is of great importance, since their leakage or loss of control, even at a very low concentration, may lead to extensive hazards to human health. ${ }^{1-3}$. Furthermore, there is a pressing need for detection of amines in our daily life, like assessment of food quality, pollution control, and management of some diseases. ${ }^{4}$ Recently, various techniques were developed and applied for the detection of hazardous amines. Partciularly, luminescence-based sensing approaches gained substantial attention due to their simple handling procedures, high sensitivity, and fast response time. ${ }^{5,6}$ While different groups have reported the sensing of amines, their discriminative sensing within a vast group of chemicals remains scarce due to challenges associated with the molecular identification of amines. ${ }^{7,8}$ Accordingly, the amines' structural, chemical and physical characteristics must be considered, like their molecular size, shapes, hydrogen bond formation capability and electronic properties. ${ }^{9-12}$

Chemical sensors are designed to identify a probe-analyte via a change in their properties, e.g., their conductivity, color, luminescence, or capacitance. ${ }^{13}$ Most of the sensing of such analytes is based on fluorescence quenching. ${ }^{14-15}$ Examples of fluorescence-intensity enhancement, i.e., a "turn-on" behavior for sensing of amines, are scarce; although, they are more desirable for use in sensing devices. ${ }^{16}$ Notably, the ability to construct a sensor with a "turn-on" feature is still a challenge, due to many characteristics that confine and obstruct with fluorescence phenomena in general.
Recently, metal-organic frameworks (MOFs) have been widely explored as sensors as a result of their fast, reversible, and durable sensing features. ${ }^{13,17-19}$ MOFs, which are inorganic-organic hybrid crystalline materials, offer diverse structural features with tunable pore shape, dimensions and functionalities..$^{20-25}$ The modularity of the MOF pore system offers the ability to decoarate the pores with a periodic array of chemical functionalities, governing molecularlevel interactions between the framework and probe analytes and affording the selective sensing toward certain analytes. ${ }^{13,17}$ Strategically, it is very thrilling to design and synthesize MOFs for discriminative "turn on" recognition of amines through interactions between an electron-deficient ligand and electron-rich amines, which can perturb the MOF photoluminescence (PL). Different from recent reports on MOFs used to sense organic amines, ${ }^{7,8}$ we investigated an interesting MOF that offers a turn-on sensing behavior for aliphatic amine.

Here, we successfully synthesized a luminescent $\mathrm{Zr}(\mathrm{IV})$-based MOF, Zr-BTDB-fcu-MOF (1), with the underlying fcu topology, by incorporating a $\pi$-conjugated, electron-deficient, thiadiazolefunctionalized ligand $\mathrm{H}_{2} \mathrm{BTDB} \quad\left\{\mathrm{H}_{2} \mathrm{BTDB}=4,4{ }^{\prime}\right.$ (benzoic]i1,2,5]thiadiazole-4,7-diyl) dibenzoic acid $\}$ (see Figure 1a and Figures S1-S5 in the Supporting Information ). ${ }^{26}$ Notably, few $\mathrm{Zr}(\mathrm{IV})$-based MOF materials containing a benzothiadiazole moiety with excellent PL properties have been demonstrated in the literature. ${ }^{27-29}$ The synthesized (1) structure was confirmed using powder X-ray diffraction (PXRD) (Figure S6). ${ }^{28}$ The synthesized (1) is a UiO-68 type and has two type of cages, a big one $(\sim 25 \AA)$ and smal one $(\sim 15 \AA)$ in pore diameter that are connected via triangular window with diameter $\sim 10 \AA . \mathrm{N}_{2}$ sorption measurements at $77 \mathrm{~K}$ of (1) revealed a type (I) reversible isotherm and an apparent BET surface area of $2380 \mathrm{~m}^{2}$ $\mathrm{g}^{-1}$ (Figure S8). The chemical stability of (1) in various solvents was confirmed by PXRD and adsorption (Figure S6). Thermogravimetric analysis showed that (1) is stable up to $350{ }^{\circ} \mathrm{C}$ in nitrogen atmosphere (Figure S9).

Appropriately, (1) displayed strong absorption band at $385 \mathrm{~nm}$ in aqueous medium (Figure S10). Luminescence of (1) and free ligand, has been thoroughly examined upon excitation at $385 \mathrm{~nm}$ as shown in Figure 1b. In water, (1) unveils a broad emission band with a $\lambda_{\max }$ at $501 \mathrm{~nm}$, which is redshifted by $20 \mathrm{~nm}$ compared to free $\mathrm{H}_{2} \mathrm{BTDB}$. This emission band was ascribed to $\pi-\pi *$ transition of organic ligand in (1). ${ }^{31}$

The luminescence-sensing studies were carried on the following VOAs: methylamine (MA), ethylamine (EA), triethylamine (TEA), aniline (A), and para-phenylenediamine (PDA) (Figure 2, Figure S11-S17 in SI). In order to gain better insights on structure-property relationship, we deployed a 
(a)

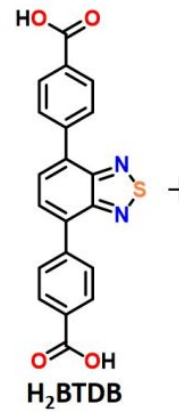

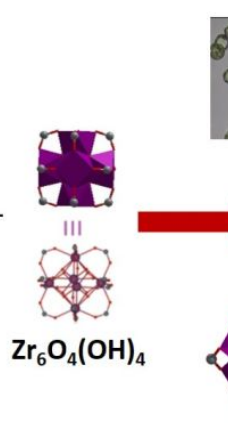

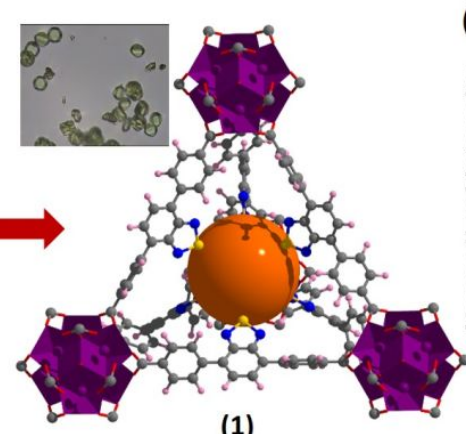

(1)

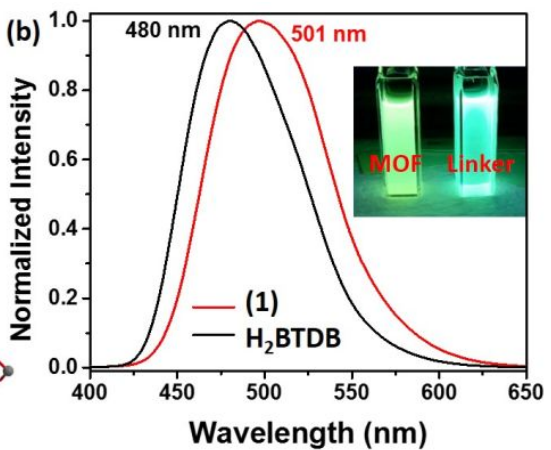

Figure. 1. (a) Schematic representation of the synthesis of Zr-BTDB-fcu-MOF (1), (inset) optical images of (1) single crystals. (b) PL spectra for (1) (red) and linker (black). (Inset) Optical images of luminescence for MOF and linker solutions under $365 \mathrm{~nm}$ UV radiation.

systematic protocol for all sensing experiments. From each aqueous solution of VOAs, portions of a $3 \mu \mathrm{M}$ were added in an incremental manner to an aqueous well-dispersed suspension of (1). Subsequently, fluorescence intensity of (1) was recorded after each incremental addition.

Notably, increased concentrations of aromatic amines, like aniline and phenylenediamine, led to a drastic decrease in fluorescence intensity of (1) (Figure $2 \mathrm{~b}$ and S16), suggesting an electron transfer process that can also quench fluorescence. However, due to the absence of spectral overlap between emission of the linker and absorption of aniline, the energy transfer can be discarded here. Alternatively, in case of aliphatic amines like MA, the fluorescence intensity was enhanced. Whereas in the case of pyridine $\left(\mathrm{pk}_{\mathrm{a}}=3.2\right)$, no change in the fluorescence intensity was observed (Figure S18). Among all VOAs tested, MA displayed the highest turn-on efficiency. These observations suggest that (1) can discriminately sense aliphatic amines via a "turn-on" fluorescence behavior (Figure 2a). Furthermore, the detection limit of (1) towards MA was determined to be $66.2 \mathrm{nM}$ according to the equation $3 \sigma / \mathrm{k}^{31,32}$ These results indicate that (1) is more sensitive toward MA than other tested amines. Interestingly, we observed that turn-on detection only occurred in aqueous media; i.e. when we carried out same set of experiments in anhydrous acetonitrile and absolute ethanol, no change was observed upon addition of MA (Figure S19 in SI), signifying the importance of protonation for sensing.

Figure 2c shows time-resolved PL lifetime (TRPL) measurements. Linker emission in (1) shows a bi-exponential decay with lifetimes of $1.8 \mathrm{~ns}(62 \%)$ and $8.9 \mathrm{~ns}(38 \%)$. When aniline was added, the lifetimes became shorter, reaching 1 ns $(80 \%)$ and $6.5 \mathrm{~ns}(20 \%)$, consistent with the emission quenching observed in steady-state measurements. In contrast, when MA was added, emissions showed a single exponential decay of 11.5 ns. In contrast to earlier literature reports, (1) is one of the exceptional examples that can discriminately sense aliphatic amines in a "turn-on" process. In order to gain insight on the sensing mechanism governing the turn-on behavior of (1) in response to aliphatic amines, we elaborated on our examination study. In this case, if our assumption is correct, then this increase in fluorescence intensity is directly related to basicity of amines. We tried to understand whether the sensitivity of (1) was due to amine structure or to a change in $\mathrm{pH}$ of the media caused by the addition of amine. Therefore, we collected PL spectra of (1) in buffer solutions with different $\mathrm{pH}$ values $(\mathrm{pH} 2, \mathrm{pH} 3, \mathrm{pH} 7, \mathrm{pH} 8$, $\mathrm{pH} 9$, and $\mathrm{pH} 10$ ). Interestingly, only a negligible enhancement in fluorescence intensity was observed (Figure S20 in SI), corroborating that the change in sensitivity was not related to a change in $\mathrm{pH}$. Here, we assume that the selected VOAs can easily diffuse in (1), due to its relatively larger pore-aperture size, affording the hosted VOA molecules to interact with exposed surface of (1) pore system. It should be noted that the diameters of tested VOAs are smaller than the pore-aperture size of the triangular sole entrance $(\sim 10 \AA)$ to octahedral and tetrahedral cages of (1). ${ }^{33}$

Perceptibly, we designed a series of additional experiments to support these facts and to understand the plausible interactions between the analyte and the thiadiazole core of the linker. We performed similar experiments with an isoreticular MOF (2) (Figures $3 \mathrm{a}$ and S7), bearing an anthracene core instead of thiadiazole (Figure $3 \mathrm{~b}$ and Figure S21). Distinctively, no significant change in fluorescence intensity was observed when MA was added to solution containing (2), confirming that aliphatic amines had strong interaction with the thiadiazole core. Therefore, we tried to understand the mode of interaction between aliphatic amine and thiadiazole core. In general, aliphatic amines are prone to protonation in water due to their high $\mathrm{pk}_{\mathrm{a}}$ values (e.g., $\left.\mathrm{pk}_{\mathrm{a}} \mathrm{MAH}^{+}=10.6, \mathrm{pk}_{\mathrm{a}} \mathrm{TEAH}^{+}=11.1\right) .{ }^{34}$ Note, aromatic amines are
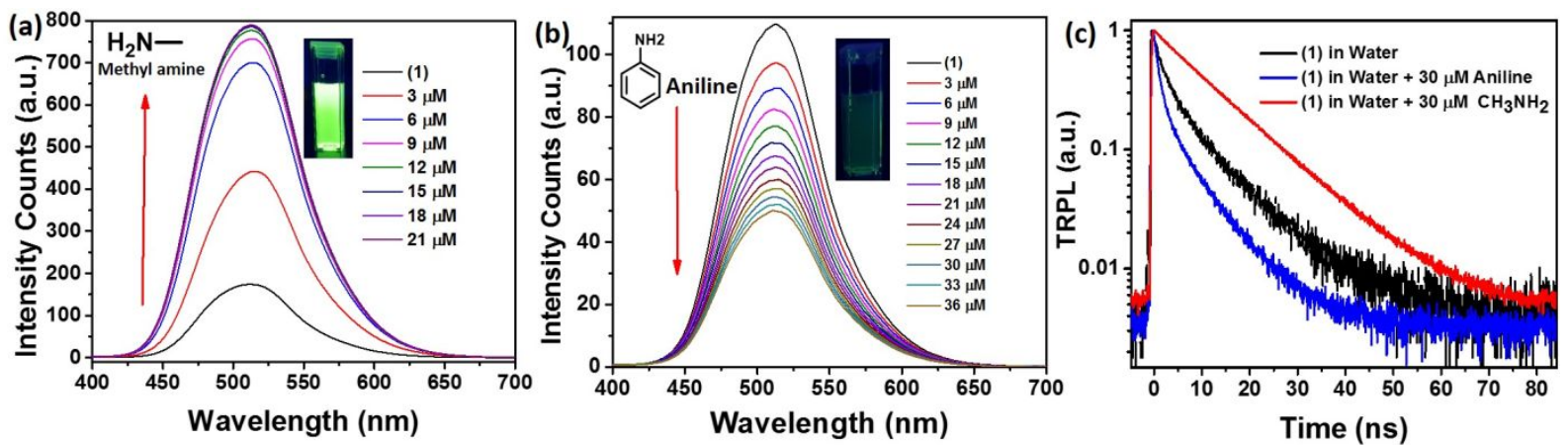

Figure 2. Fluorescence intensity of (1) aqueous suspension upon addition of $3 \mu \mathrm{M}$ of MA (a), aniline (b) $\left(\lambda_{\max }=515 \mathrm{~nm}\right)$. c) Fluorescence lifetime decay profile of (1) before and after addition of $225 \mu \mathrm{L} \mathrm{MA}\left(\lambda_{\mathrm{ex}}=400 \mathrm{~nm}\right)$. 

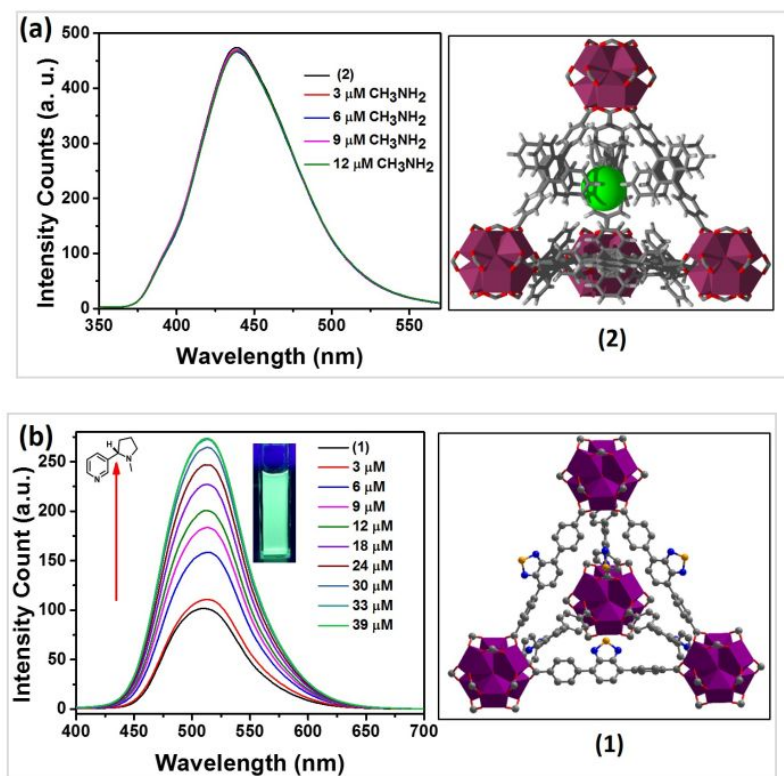

Figure 3. (a) Fluorescence intensity of (2) water suspension upon addition of $3 \mu \mathrm{M}$ MA $\left(\lambda_{\max }=447 \mathrm{~nm}\right)$. (b) The increase in Fluorescent of (1) upon addition of $3 \mu \mathrm{M}$ nicotine and its chemical structure.

not protonated in water at $\mathrm{pH}=7.0$. We also conducted control experiment with the linker alone, and the results indicate less sensitivity compared with (1) (Figure 25). To understand the effect of protonated MA on PL enhancement of $\mathrm{H}_{2} \mathrm{BTDB}$, we performed density functional theory (DFT) calculations, as illustrated in Figure 4. For $\mathrm{H}_{2} \mathrm{BTDB}$ alone, the dihedral angle between benzene and thiadiazole unit changes significantly from ground to excited state $\left(\Delta=22^{\circ}\right)$, increasing the

non-radiative channels. While once two or four MA molecules are attached to $\mathrm{H}_{2} \mathrm{BTDB}$, the change of dihedral angle becomes considerably smaller $\left(\Delta=12^{\circ}\right)$, due to the strong hydrogen bonding $\left(d_{\mathrm{H}}=1.9 \AA\right)$ between protonated $\mathrm{MA}$ and $\mathrm{N}$ atoms on thiadiazole unit. This demonstrates clearly that the H-bonding interaction restricts the rotation of the thiadiazole core, reducing non-radiative recombination pathways and subsequently enhancing emission intensity. Considerately, we calculated the ratio between MA and thiadiazole linker (L) in (1). The ratio of 2:1 (MA: L) was found; and reaches almost saturation upon addition of $12 \mu \mathrm{M}$. For instance, increasing the concentration to $21 \mathrm{mM}$, which is still below the ratio 4:1, does not change the fluorescence enhancement, which is in excellent agreement with DFT calculations..$^{35}$

Additionally, we measured the solid-state NMR of (1) and the linker (Figure S26) where a chemical shift for the $-\mathrm{COOH}$ proton at $\sim 15 \mathrm{ppm}$ (in linker) was missing from the MOF due to coordination with $\mathrm{Zr}(\mathrm{IV})$, and no additional $\mathrm{N}-\mathrm{H}$ protons were observed. These results confirm that the thiadiazole core was not protonated during the synthesis and that it can easily participate in formation of a hydrogen bond with incoming protonated amines. Furthermore, we collected PL spectra from (1) in presence of $\mathrm{NH}_{4} \mathrm{Cl}$. The PL spectra showed a turn-on fluorescence in aqueous media due to hydrogen-bonding interaction with $\mathrm{NH}_{4}{ }^{+}$moiety (Figure S22). By adding $\mathrm{NH}_{4} \mathrm{Cl}$ in presence of an amine into (1) aqueous solution, the fluorescence intensity was enhanced (Figure S23), indicating that the interaction of $\mathrm{NH}_{4}{ }^{+}$with the framework was stronger than its interaction with the protonated amine. It also confirms that the formation of hydrogen bond with linker was the key force restricting the rotation of thiadiazole group and facilitating the extended $\pi$ conjugation, thus resulting in the turnon fluorescence behavior (Figure 3). This turn-on sensing of an aliphatic amine by (1) is also transferable to other materials, such as nicotine. Nicotine is an extremely harmful compound present in tobacco products, which make it very attractive to detect even at low concentrations in aqueous (or biological) medium. Nicotine contains an aliphatic amine functionality with a $\mathrm{pk}_{\mathrm{a}}$ of 7.9. Therefore, a low concentration could be detected in an aqueous medium with a similar turn-on fluorescence sensing. The detection limit of (1) for nicotine was calculated to be around 280 $\mathrm{nM}$ (Figure 4 and S24).

In conclusion, we have successfully explored the sensing properties of a highly luminescent $\mathrm{Zr}$ (IV)-based MOF material (1), towards aliphatic amines. Delightfully, (1) exhibits a selective detection behavior towards aliphatic amines (via fluorescence turn-on behavior), as established from PL titration measurements. The mechanism of sensing via hydrogen bonding was validated experimentally and supported by DFT calculations. (1) exhibited a detection limit of $66.2 \mathrm{nM}$ for MA, which is considered superior to some previously reported MOF-based sensors. Given its excellent detection performance as well as its high thermal and chemical stability, Zr-BTDB-fcu-MOF (1) offers great prospects as a sensor for environmental applications.

\section{ASSOCIATED CONTENT}

\section{Supporting Information}

The Supporting Information is available free of charge on the ACS Publications website. Synthesis, experimental data and procedures, X-ray diffraction, sorption data and photoluminescence measurements

\section{AUTHOR INFORMATION}

\section{Corresponding Author}

Mohamed Eddaoudi: omar.abdelsaboor@kaust.edu.sa (a)

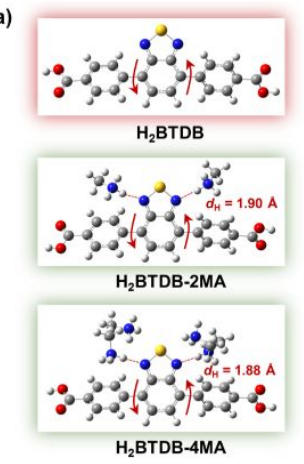

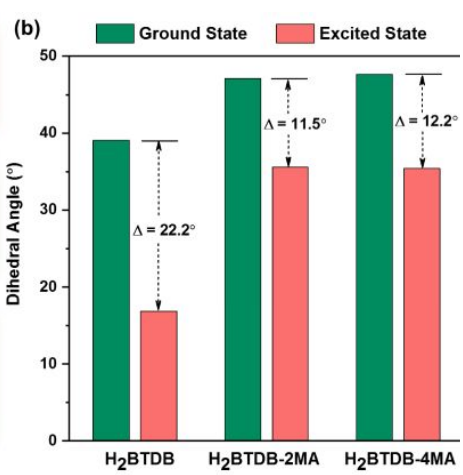

Figure 4. (a) Molecular structures of $\mathrm{H}_{2} \mathrm{BTDB}, \mathrm{H}_{2} \mathrm{BTDB}$ with two and four MA molecules. Red arrows represent the dihedral angles between benzene and thiadiazole-based units and red dashed lines indicate the hydrogen bond lengths $\left(d_{\mathrm{H}}\right)$ formed by $\mathrm{H}$ of MA and $\mathrm{N}$ of thiadiazole; (b) Dihedral angles between benzene and thiadiazole units for ground and excited states of $\mathrm{H}_{2} \mathrm{BTDB}, \mathrm{H}_{2} \mathrm{BTDB}$ with two and four MA molecules. $\Delta$ represents the dihedral angle change from ground to excited state. The calculations are performed in water using CAM-B3LYP/6-31(d,p) method.

Omar F. Mohammed: mohamed.eddaoudi@kaust.edu.sa

Notes

The authors declare no competing financial interests.

\section{ACKNOWLEDGMENT}


The authors gratefully acknowledge financial support from King Abdullah University of Science and Technology (KAUST). In the memory of Abdallah Eddaoudi

\section{REFERENCES}

(1) Merchant, Z. M.; Cheng, S. G. G. In Characterization of Foods, Emerging Methods ; Gaonkar, A. G., Ed.; Elsevier Science: New York, 1995; Chapter 15.

(2) Ajayakumar, M. R.; Mukhopadhyay, P. Naphthalene-bishydrazimide: radical anions and ICT as new bimodal probes for differential sensing of a library of amines. Chem. Commun. 2009, 25, 3702-3704

(3) Bao, B.; Yuwen, L.; Zheng, X.; Weng, L.; Zhu, X.; Zhan, X.; Wang, L. A fluorescent conjugated polymer for trace detection of diamines and biogenic polyamines. J. Mater. Chem. 2010, 20, 9628-9634.

(4) Medina, M. Á.; Urdiales, J. L.; Rodríguez-Caso, C.; Ramírez F. J.; Sánchez-Jiménez, F. Biogenic amines and polyamines: similar biochemistry for different physiological missions and biomedical applications. Crit. Rev. Biochem. Mol. Biol., 2003, 38, 23.

(5) Hinoue, T.; Miyata, M.; Hisaki, I.; Tohnai, N. Guest-Responsive Fluorescence of Inclusion Crystals with n-Stacked Supra-molecular Beads. Angew. Chem., Int. Ed. 2012, 51, 155-158.

(6) Stetter, J. R.; Penrose, W. R.; Yao, S. Sensors, Chemical Sensors, Electrochemical Sensors, and ECS. J. Electrochem. Soc. 2003, 150, S11-S16.

(7) Liu, J. J.; Shan, Y. B.; Fan, C. R.; Lin, M. J.; Huang, C. C.; Dai, W. $X$. Encapsulating Naphthalene in an Electron-Deficient MOF to Enhance Fluorescence for Organic Amines Sensing. Inorg. Chem. 2016, 55, 3680-3684.

(8) Mallick, A.; Garai, B.; Addicoat, M. A.; Petkov, P., St.; Heine, T.; Banerjee, R. Solid state organic amine detection in a photochromic porous metal organic framework. Chem. Sci. 2015, 6, 1420-1425.

(9) Mertz, E.; Zimmerman, S. C. Cross-Linked Dendrimer Hosts Containing Reporter Groups for Amine Guests. J. Am. Chem. Soc. 2003 , $125,3424-3425$.

(10) Greene, N. T.; Morgan, S. L.; Shimizu, K. D. Molecularly imprinted polymer sensor arrays. Chem. Commun. 2004, 1172-1173.

(11) Comes, M.; Marcos, M. D.; Martınez-Manez, R.; Sancenon, F.; Soto, J.; Villaescusa, L. A.; Amoros, P.; Beltran, D. Chromogenic Discrimination of Primary Aliphatic Amines in Water with Functionalized Mesoporous Silica. Adv. Mater. 2004, 16, 1783-1786.

(12) Mohr, G. J. Chromo- and Fluororeactands: Indicators for Detection of Neutral Analytes by Using Reversible Covalent-Bond Chemistry. Chem. - Eur. J. 2004, 10, 1082-1090. (b) Chernikova, V.; Yassine, O.; Shekhah, O.; Eddaoudi, M.; Salama, K. N. Highly Sensitive and Selective SO2 MOF Sensor: the Integration of MFM-300 MOF as a Sensitive Layer on a Capacitive Interdigitated Electrode. J. Mater. Chem. A, 2018, 6, 5550.

(13) Kreno, L. E.; Leong, K.; Farha, O. K.; Allendorf, M.; Van Duyne, R. P.; Hupp, J. T. Metal-Organic Framework Materials as Chemical Sensors. Chem. Rev. 2012, 112, 1105-1125.

(14) Che, Y.; Zang, L. Enhanced fluorescence sensing of amine vapor based on ultrathin nanofibers. Chem. Commun. 2009, 34, 5106-5108.

(15) Takashima, Y.; Martínez, V. M.; Furukawa, S.; Kondo, M.; Shimomura, S.; Uehara, H.; Nakahama, M.; Sugimoto, K.; Kitagawa, S. Molecular decoding using luminescence from an entangled porous framework. Nat. Commun. 2011, 2, 168.

(16) Kumpf, J.; Freudenberg, J.; Fletcher, K.; Dreuw, A.; Bunz, U. H. F. Detection of Amines with Extended Distyrylbenzenes by Strip Assays. J. Org. Chem. 2014, 79, 6634-6645.

(17) Pramanik, S.; Zheng, C.; Zhang, X.; Emge, T. J.; Li, J. New Microporous Metal-Organic Framework Demonstrating Unique Selectivity for Detection of High Explosives and Aromatic Compounds. $J$. Am. Chem. Soc. 2011, 133, 4153-1455.

(18) (a) Gole, B.; Bar, A. K.; Mukherjee, P. S. Fluorescent metal-organic framework for selective sensing of nitroaromatic explosives. Chem. Commun. 2011, 47, 12137-12139. (b) Wang, J. H.; Li, M.; Li, D. A dynamic, luminescent and entangled MOF as a qualitative sensor for volatile organic solvents and a quantitative monitor for acetonitrile vapour. Chem. Sci. 2013, 4, 1793-1801.
(19) Asha, K. S.; Bhattacharyya, K.; Mandal, S. Discriminative detection of nitro aromatic explosives by a luminescent metal-organic framework. J. Mater. Chem. C 2014, 2, 10073-10081.

(20) Yaghi, O. M.; Li, H.; Eddaoudi, M.; O’ Keeffe, M. Design and synthesis of an exceptionally stable and highly porous metal-organic framework. Nature, 1999, 402, 276-279.

(21) Deng, H.; Grunder, S.; Cordova, K. E.; Valente, C.; Furukawa, H.; Hmadeh, M.; Gandara, F.; Whalley, A. C.; Liu, Z.; Asahina, S.; Kazumori, H.; O'Keeffe, M.; Terasaki, O.; Stoddart, J. F.; Yaghi, O. M. Large-Pore Apertures in a Series of Metal-Organic Frameworks. Science, 2012, 336, $1018-1023$.

(22) Huang, R. W.; Wei, Y. S.; Dong, X. Y.; Wu, X. H.; Du, C. X.; Zang, S. Q.; Mak, T. C. W. Hypersensitive dual-function luminescence switching of a silver-chalcogenolate cluster-based metal-organic framework. Nat. Chem. 2017, 9, 689-697.

(23) Bloch, W. M.; Burgun, A.; Coghlan, C. J.; Lee, R.; Coote, M. L.; Doonan, C. J.; Sumby, C. J. Capturing snapshots of post-synthetic metallation chemistry in metal-organic frameworks. Nat. Chem. 2014, 6, 906-912.

(24) Van-Humbeck, J. F.; McDonald, T. M.; Jing, X.; Wiers, B. M.; Zhu, G.; Long, J. R. Ammonia Capture in Porous Organic Polymers Densely Functionalized with Brønsted Acid Groups. J. Am. Chem. Soc. 2014, 136, 2432-2440.

(25) Deria, P.; Bury, W.; Hupp, J. T.; Farha, O. K. Versatile Functionalization of the NU-1000 Platform by Solvent-assisted Ligand Incorporation. Chem. Commun. 2014, 50, 1965-1968.

(26) Mostakim, SK.; Biswas, S. A Thiadiazole-functionalized Zr(IV)based Metal-Organic Framework as a Highly Fluorescent Probe for the Selective Detection of Picric Acid. CrystEngComm, 2016, 18, 3104-3113.

(27) Zhang, X.; Gorohmaru, H.; Kadowaki, M.; Kobayashi, T. Ishi-i, T.; Thiemann, T. Mataka, S. Benzo-2,1,3-thiadiazole-based, Highly Dichroic Fluorescent Dyes for Fluorescent Host-Guest Liquid Crystal Displays. J. Mater. Chem., 2004, 14, 1901-1904.

(28) Dong, Y.; Koken, B.; Ma, X.; Wang, L.; Cheng, Y.; Zhu, C.; Polymer-based Fluorescent Sensor Incorporating 2,2'-bipyridyl and Benzo[2,1,3]thiadiazole Moieties for $\mathrm{Cu}^{2+}$ Detection. Inorg. Chem. Commun., 2011, 14, 1719-1722.

(29) Edelmann, M. J.; Raimundo, J.-M.; Utesch, N. F.; Diederich, F.; Dramatically Enhanced Fluorescence of Heteroaromatic Chromophores upon Insertion as Spacers into Oligo(triacetylne)s. Helv. Chim. Acta, 2002, 85, 2195-2213.

(30) Zhang, W.-Q.; Li, Q.-Y.; Cheng, J.-Y.; Cheng, K.; Yang, X.; Li, Y.; Zhao, X.; Wang, X.-J. Ratiometric Luminescent Detection of Organic Amines Due to the Induced Lactam-Lactim Tautomerization of Organic Linker in a Metal-Organic Framework. ACS Appl. Mater. Interfaces, 2017, 9, 31352-31356.

(31) Hussain, S.; De, S.; Iyer, P. K. Thiazole-Containing Conjugated Polymer as a Visual and Fluorometric Sensor for Iodide and Mercury. ACS Appl. Mater. Interfaces, 2013, 5, 2234-2240.

(32) Masih, D.; Chernikova, V.; Shekhah, O.; Eddaoudi, M.; Mohammed, O. F. Zeolite-Like Metal-Organic Framework (MOF) Encaged Pt(II)-Porphyrin for Anion-Selective Sensing. ACS Appl. Mater. Interfaces, 2018, 10, 11399-11405.

(33) Schaate, A.; Roy, P.; Godt, A.; Lippke, J.; Waltz, F.; Wiebcke, M.; Behrens, P. Modulated Synthesis of Zr-Based Metal-Organic Frameworks: From Nano to Single Crystals. Chem. Eur. J., 2011, 17, 6643-6651.

(34) Hall Jr., H.K. Sterically Hindered Phenolic Buffers. Application to Determination of Rates of Amidation of Ethyl Chloroformate. J. A.m. Chem. Soc. 1957, 79, 5439-5441.

(35) El-Zohry, A. M.; Alturki, A.; Yin, J.; Mallick, A.; Shekhah, O.; Eddaoudi, M.; Ooi, B. S.; Mohammed, O. F. Tunable Twisting Motion of Organic Linkers via Concentration and Hydrogen-Bond Formation $J$. Phys. Chem. C 2019, 123, 5900-5906. 


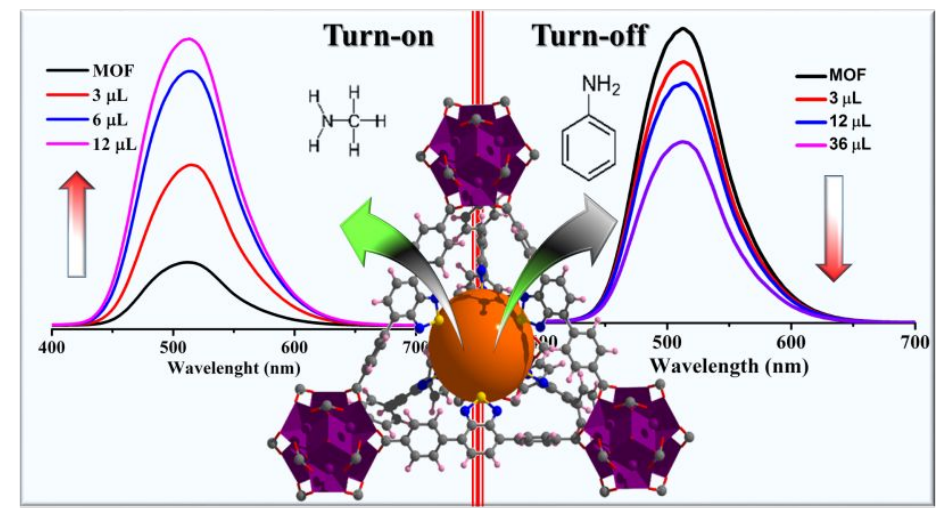

